Une banque de données commune à l'Office fédéral de la santé publique (OFSP), aux organisations professionnelles et aux autorités sanitaires cantonales

\title{
Le registre des professions médicales (registre)
}

Maria Hodel

Co-Cheffe Section Formation des professions médicales Co-Cheffe du Service Registre des professions médicales
Correspondance:

Maria Hodel

Département fédéral

de l'intérieur DFI

Office fédéral de la santé

publique OFSP

Unité de direction

Politique de la santé

Schwarzenburgstrasse 161

CH-3007 Berne

Tél. 0313238787

Fax 0313238805

maria.hodel@bag.admin.ch

www.bag.admin.ch
Ces deux dernières années, l'OFSP a développé un registre en ligne, relatif au personnel médical universitaire. Le registre comptait au nombre des projets qu'exigeait la loi fédérale sur les professions médicales universitaires (LPMéd). Cette dernière ainsi que les ordonnances y afférentes réglementent la formation et la formation postgrade ainsi que l'exercice indépendant des professions dans les cinq domaines suivants: médecine humaine, médecine dentaire, médecine vétérinaire, pharmacie et chiropratique. L'ordonnance sur le registre est entrée en vigueur le $1^{\mathrm{er}}$ novembre 2008.

Objectif de cette nouvelle banque de données Le législateur cite l'information et la protection des patients comme but premier du registre. La mobilité croissante du personnel médical et des médecins à l'intérieur de la Suisse et au sein de l'espace européen notamment, place les autorités cantonales chargées de l'octroi des autorisations face à d'importants défis. Il s'agit de la première banque de données centrale et supracantonale qui contienne des informations détaillées concernant les qualifications professionnelles et les autorisations cantonales d'exercer pour les professions mentionnées ci-dessus. Cette nouvelle banque de données en ligne propose aux autorités cantonales délivrant les autorisations des données personnelles standardisées ainsi que des données fiables concernant les diplômes et titres de formation postgrade. L'accès simple aux autorisations d'exercer la profession de tous les cantons ainsi qu'aux données personnelles particulièrement sensibles (p.ex. mesures disciplinaires) augmentent la transparence et la sécurité. Agissant comme plaque tournante, l'OFSP recueille ces données et les met à la disposition des services cantonaux habilités, lorsque ceux-ci en font la demande. Cette mesure doit permettre de simplifier la procédure pour l'octroi des autorisations cantonales d'exercer.

\section{Travaux actuels}

L'OFSP a réuni les données relatives au personnel médical provenant de sa propre banque de données, qui recense les personnes titulaires de diplômes fédéraux ou de diplômes étrangers reconnus, avec les données provenant de toutes les banques de données des organisations professionnelles et de santésuisse. Les autorités cantonales délivrant les autorisations disposent désormais d'une base de données aussi complète que possible. D'ici la fin 2009, l'OFSP, les organisations professionnelles et les autorités sanitaires cantonales examineront et complèteront les informations concernant les près de 60000 membres du personnel médical. Ceci afin d'obtenir des données d'une qualité optimale. Conformément à l'ordonnance sur le registre, le public intéressé pourra, au $1^{\mathrm{er}}$ janvier 2010, accéder à la banque de données, via l'adresse Internet www. medreg.admin.ch

\section{Quelles données le registre contient-il?}

Le registre contient les données personnelles ainsi que tous les diplômes et titres postgrades fédéraux ou étrangers reconnus. Dès à présent, chaque praticien se voit attribuer, lorsqu'il reçoit son diplôme ou la reconnaissance pour celui-ci (membres UE/AELE), le numéro GLN (Global Location Number) grâce auquel il s'identifiera et qui l'accompagnera dans l'exercice de la profession. La Fédération des médecins suisses (FMH) enregistrera dans cette banque de données également les diplômes postgrades de droit privé qu'elle délivre (spécialisation, domaines de prédilection, certificats de capacité). Le registre ne contiendra pas d'informations concernant d'autres qualifications professionnelles ou académiques, ou sur des domaines d'activité spécifiques. Les autorités cantonales délivrant les autorisations inscrivent les données concernant les autorisations de pratiquer. Au nombre de ces données, on compte les informations relatives à la gestion d'une pharmacie privée ainsi que les droits concernant l'utilisation de stupéfiants.

De plus, l'OFSP et les autorités cantonales peuvent, en consultant le registre, savoir si une autorité cantonale délivrant des autorisations a pris des mesures disciplinaires (définitives) contre un praticien (p.ex. avertissement, retrait de durée limitée ou illimitée de l'autorisation d'exercer). Si l'OFSP dispose d'informations concernant des mesures disciplinaires prises envers des médecins de l'UE qui ont demandé une reconnaissance de leur diplôme en Suisse, il peut également intégrer ces données dans le registre. Seuls les retraits d'autorisation pour non-satisfaction des exigences ou les interdictions de pratiquer seront accessibles au public. Dans ce cas, l'information selon laquelle la personne concernée n'a pas d'autorisation est publiée. Les mesures mêmes ainsi que les motifs ne sont pas consignés dans le registre. Seuls l'OFSP et les autorités cantonales délivrant les autorisations y ont accès.

Une liste détaillée de toutes les données ainsi que des droits et des devoirs des partenaires impliqués se 
trouve dans l'ordonnance sur le registre www.admin. ch/ch/f/rs/c811_117_3.html

\section{Qu'est-ce que le GLN?}

Le Global Location Number (GLN) est la nouvelle désignation du European Article Number (EAN). Il est attribué par l'OFSP à tout praticien lorsqu'il reçoit son diplôme ou la reconnaissance pour celui-ci et lui servira d'identifiant. Les EAN déjà attribués sont repris et restent valables.

\section{Nouvelle compétence pour l'attribution du numéro GLN}

Dès à présent, tout praticien se voit attribuer un GLN par l'OFSP lorsqu'il reçoit son diplôme fédéral. L'attribution du GLN se fait également simultanément à la reconnaissance de diplômes provenant des Etats membres de l'UE/AELE ou à l'établissement d'une attestation d'équivalence pour les titulaires de diplômes de pays non-membres de l'UE (art. 36, al. 3, LPMéd). Le GLN figure désormais sur le diplôme et sur le titre postgrade; il est également publié dans le registre. La dénomination GLN est la nouvelle désignation pour l'EAN (European Article Number). Le GLN doit être mentionné dans toute communication et toute correspondance (papier à lettres, factures, page d'accueil). Il apparaît désormais au nombre des références sur le diplôme fédéral ou reconnu de chaque praticien.

Nous recommandons aux médecins ressortissants de l'UE/AELE qui ont besoin d'un GLN d'effectuer la procédure de reconnaissance, même si aucune raison

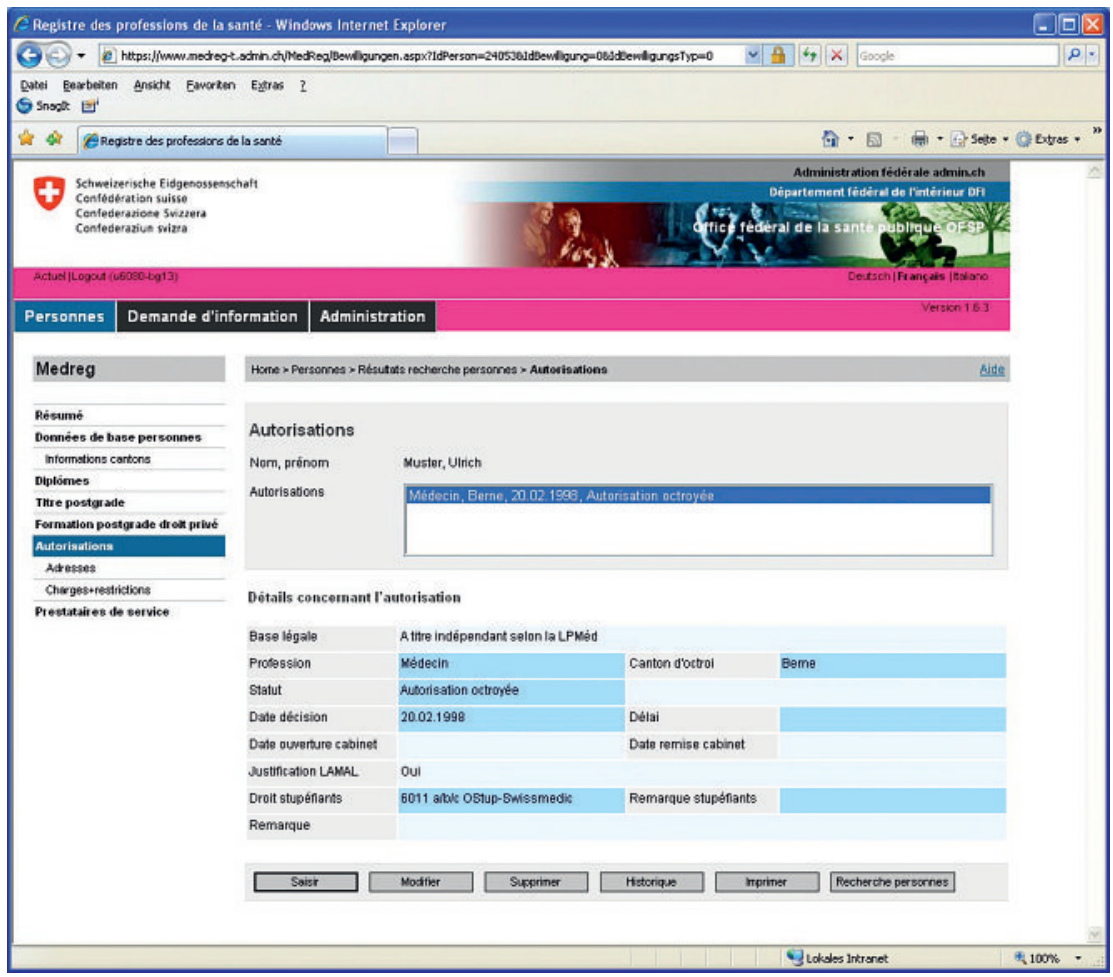

Le MedReg offre à tous les partenaires impliqués et au public des informations transparentes sur les personnes relevantes des professions médicales universitaires et contribue ainsi à la protection des patients. légale ne les oblige à faire reconnaître leur diplôme (p.ex. médecins assistants). Et ce pour la raison suivante: l'attribution d'un titre postgrade fédéral n'est possible que si le diplôme a été reconnu au préalable. Les médecins en provenance de pays non-membres de l'UE/AELE qui ont besoin d'un GLN continuent de déposer une demande auprès de la FMH.

\section{Droits et devoirs de la FMH}

La FMH - ou plus généralement les organisations professionnelles des cinq professions médicales universitaires - est tenue, conformément à la LPMéd, d'inscrire les titres postgrades fédéraux dans le registre. En revanche, l'inscription des qualifications de droit privé (titre postgrade, domaines de prédilection, certificats de capacité FMH) est facultative. Cette possibilité a été précisée dans l'ordonnance sur le registre à la demande des organisations professionnelles. L'OFSP est d'avis qu'une liste complète de toutes les qualifications sert non seulement à l'information du patient mais qu'elle est également dans l'intérêt du médecin. De fait, la FMH publiera dans le registre l'ensemble des diplômes postgrades de droit privé.

L'avantage du registre pour la FMH est que celle-ci peut intégrer à sa propre banque de données toutes les informations sur les nouveaux praticiens titulaires d'un diplôme fédéral ou étranger reconnu qu'elle aura trouvées dans le registre.

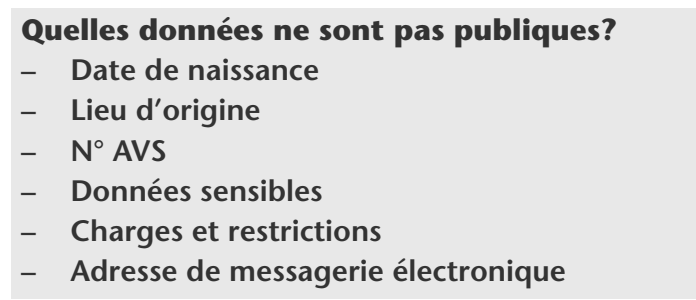

\section{Vos droits et devoirs en tant que médecin}

Conformément à la LPMéd et à l'ordonnance sur le registre, vous n'êtes pas tenu d'intégrer les données directement dans le registre. Ces données sont enregistrées par l'OFSP, la FMH et les autorités cantonales, conformément aux compétences prévues dans l'ordonnance sur le registre. En tant que médecin et dans le cas où vous exercez une activité soumise à autorisation, vous êtes toutefois tenu de vous annoncer auprès des autorités cantonales délivrant les autorisations. Certaines autorités ont à ce propos demandé aux praticiens de leur canton de les aider dans la phase actuelle de correction et d'actualisation, car certaines données les concernant sont incomplètes ou plus d'actualité. A partir du $1^{\text {er }}$ janvier 2010, vous pourrez annoncer directement dans le registre (adresse: voir ci-après) tout changement concernant votre activité professionnelle (p.ex. fermeture du cabinet, déménagement, interruption temporaire de l'activité, etc.). Les titres postgrades fédéraux et les diplômes postgrades FMH sont tenus à jour (en permanence) par la FMH. 
Que dois-je faire si mon nom n'apparaît pas sous www.medreg.admin.ch au $1^{\text {er }}$ janvier 2010?

Veuillez vous adresser par courriel à I'OFSP (medreg@bag.admin.ch).

\section{Accès au registre}

Dès à présent, vous pouvez demander un nom d'utilisateur muni d'un mot de passe à l'adresse www. medreg.admin.ch/anmelden.aspx. Les données d'accès vous seront communiquées durant le $1^{\text {er }}$ trimestre 2010. Vous pourrez ainsi consulter l'ensemble des données vous concernant et demander, par voie électro-

\section{Le MedReg offre à tous les partenaires impliqués et au public des informations transparentes sur les personnes relevantes des professions médicales universitaires et contribue ainsi à la protection des patients}

nique, une correction des données erronées ou lacunaires que vous aurez constatées. Votre requête de modification sera ensuite communiquée au service compétent. Après examen, la modification sera effectuée et tous les partenaires impliqués dans le projet (autorités cantonales délivrant les autorisations, OFSP, organisations professionnelles) en seront informés en même temps. En outre, cela permettra à la population d'avoir, p. ex., accès à la nouvelle adresse de votre cabinet. Vous avez également le droit de faire une demande auprès de l'OFSP pour consulter des données personnelles particulièrement sensibles vous concernant si tel est le cas.

Pour les membres de la FMH, un accès direct au registre est prévu via myFMH. Vous serez informés de l'entrée en fonction de cet accès en temps voulu.

\section{L'essentiel en bref}

- Le registre est une banque de données qui sert en premier lieu à informer et à protéger les patients.

- La banque de données centralisée facilite la collaboration entre les différents partenaires et augmente notamment le degré de transparence entre les autorités cantonales.

- Tous les médecins possédant une autorisation pour l'exercice indépendant de la profession doivent être inscrits dans le registre.

- De par la LPMéd, les autorités cantonales compétentes sont tenues de communiquer tout changement concernant les autorisations: octroi, refus ou modification d'une autorisation de pratiquer à titre indépendant, limitation ou mesure disciplinaire.

- Le registre des professions médicales ne constitue en aucun cas une nouvelle banque de données de référence permettant d'effectuer le décompte des prestations selon l'assurance obligatoire des soins (AOS).

- Un médecin titulaire d'une autorisation adéquate peut exercer la profession ainsi que tous les droits et devoirs que cela implique, même s'il ne figure pas dans le registre ou que son inscription comporte des lacunes (seule l'autorisation cantonale d'exercer la profession est déterminante). Il est toutefois recommandé de demander immédiatement une rectification de la situation.

Dois-je effectivement contrôler moi-même mes données dans le registre?

L'OFSP, les cantons et la FMH ont contribué avec le plus grand soin à rassembler toutes les données à leur disposition. Nous vous prions toutefois de vérifier les données vous concernant et de nous signaler d'éventuelles erreurs. Merci. 


\section{II registro delle professioni mediche (MEDREG) Una banca dati dell'Ufficio federale della sanità pubblica (UFSP) in collaborazione con le orga- nizzazioni professionali e le autorità sanitarie cantonali}

Negli ultimi due anni l'UFSP ha allestito un nuovo registro telematico sulle persone appartenenti alle professioni mediche universitarie (MEDREG). Si tratta di un progetto d'esecuzione della legge federale sulle professioni mediche universitarie (LPMed). Nella LPMed e nelle relative ordinanze sono disciplinate la formazione, il perfezionamento e il libero esercizio delle cinque professioni mediche universitarie seguenti: medico, dentista, veterinario, farmacista e chiropratico. L'ordinanza sul MEDREG è entrata in vigore il $1^{\circ}$ novembre 2008.

\section{Quali obiettivi ci si prefigge con la nuova banca dati?}

Il legislatore definisce l'informazione e la protezione dei pazienti quale primo e principale obiettivo della LPMed. L'aumento della mobilità delle persone appartenenti alle professioni mediche, e quindi anche dei medici, in Svizzera e in particolare all'interno dello spazio europeo pone le autorità cantonali preposte al rilascio delle autorizzazioni di fronte a enormi sfide. Non esisteva finora una banca dati centralizzata e sovracantonale che contenesse informazioni sulle qualifiche professionali e sulle autorizzazioni cantonali all'esercizio della professione dei succitati professionisti. La nuova banca dati telematica fornisce dati standardizzati sulla persona e dati verificati su diplomi e titoli di perfezionamento alle autorità cantonali preposte al rilascio delle autorizzazioni. Il facile accesso alle autorizzazioni all'esercizio della professione di tutti i Cantoni e ai dati sulla persona degni di particolare protezione (p.es. i provvedimenti disciplinari) accresce la trasparenza e la sicurezza. I dati sono rilevati dall'UFSP, il quale fungendo da coordinatore li mette a disposizione degli organi cantonali autorizzati che li richiedono. Ciò contribuisce alla semplificazione delle procedure di rilascio delle autorizzazioni cantonali all'esercizio della professione.

\section{Mansioni attuali}

L'UFSP ha raccolto e riunito tutti i dati sulle persone appartenenti alle professioni mediche, tratti dalla propria banca dati sui titolari di diplomi federali o di diplomi esteri riconosciuti, come anche le informazioni tratte da tutte le banche dati delle organizzazioni professionali e di santésuisse. Le autorità cantonali preposte al rilascio delle autorizzazioni dispongono in tal modo di una raccolta di dati pressoché completa. Entro la fine del 2009, l'UFSP, le organizzazioni professionali e le autorità sanitarie cantonali verificheranno e integreranno i dati sulle 60000 persone appartenenti alle professioni mediche in maniera tale da ottenere un'elevata qualità dei dati. La banca dati sarà inoltre resa accessibile al pubblico interessato dal $1^{\circ}$ gennaio 2010 all'indirizzo Internet www.medreg.admin.ch, come previsto dall'ordinanza sul registro LPMed.

\section{Quali dati contiene il MEDREG?}

Nel MEDREG sono registrati i dati personali e tutti i diplomi, rispettivamente i titoli di perfezionamento, federali o esteri riconosciuti disponibili. Ogni persona appartenente alle professioni mediche riceverà da subito, alla consegna o al riconoscimento del diploma (cittadini dell'UE o dell'AELS) il cosiddetto Global Location Number (GLN), un numero di identificazione univoco che accompagnerà il professionista dalla consegna del diploma fino alla cessazione dell'esercizio della professione. La Federazione dei medici svizzeri (FMH) immetterà nella banca dati anche i propri diplomi di perfezionamento di diritto privato (titolo di medico specialista, settori specialistici e certificati di capacità). Non saranno regi- 
strate altre qualifiche professionali o accademiche né settori di attività speciali. Spetta alle autorità cantonali preposte al rilascio delle autorizzazioni di inserire i dati attinenti all'autorizzazione cantonale all'esercizio della professione, incluse le informazioni sulla gestione di una farmacia privata e i diritti e doveri che vigono nell'utilizzazione di stupefacenti.

Attraverso il MEDREG, l'UFSP e le autorità cantonali possono inoltre controllare se una delle autorità cantonali preposte al rilascio delle autorizzazioni ha emesso un provvedimento disciplinare (con valore giuridico) a carico di una persona appartenente alle professioni mediche (ad es. un ammonimento, la revoca temporanea o a tempo indeterminato dell'autorizzazione all'esercizio della professione). Nel caso in cui l'UFSP sia a conoscenza dell'emissione di provvedimenti disciplinari nei confronti di medici provenienti da Paesi membri dell'UE che hanno fatto riconoscere in Svizzera il proprio diploma, sarà I'UFSP stesso a inserirne i dati. II pubblico può visionare le informazioni sulla revoca dell'autorizzazione solo nel caso in cui le condizioni per l'esercizio non siano state rispettate oppure sia stato pronunciato un divieto di esercizio della professione. In tal caso sarà pubblicata l'informazione sul divieto di esercizio della professione per la persona interessata. I provvedimenti stessi e le relative motivazioni non saranno pubblicati nel MEDREG. A tali informazioni hanno accesso esclusivamente l'UFSP e le autorità cantonali preposte al rilascio delle autorizzazioni.

Una lista dei dati dettagliata e completa nonché i diritti e doveri delle parti compartecipanti si trova nell'ordinanza sul registro LPMed http://www.admin.ch/ch/d/sr/c811 117 3.html

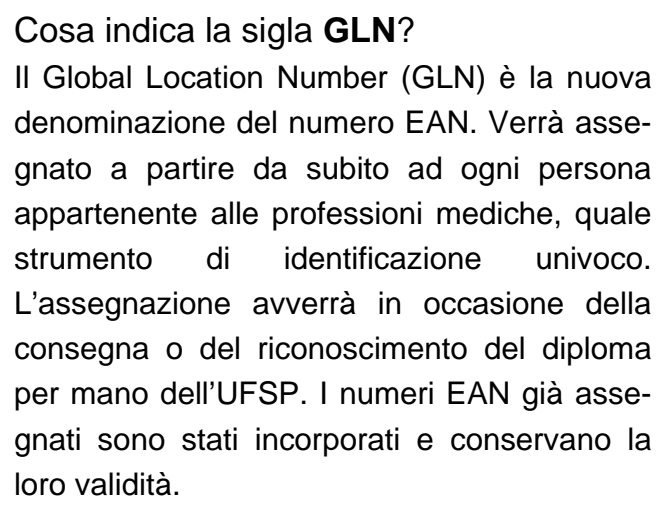

\section{Nuovo responsabile per l'assegnazione del numero GLN}

Da subito, a ogni medico verrà assegnato un numero GLN assieme al diploma federale.

L'assegnazione del numero GLN avviene anche in seguito al riconoscimento dei diplomi rilasciati dagli Stati membri dell'UE o dell'AELS oppure al momento del rilascio di un attestato di equivalenza per i titolari di un diploma rilasciato da uno Stato non membro dell'UE (art. 36 cpv. 3 LPMed). II numero GLN comparirà sul diploma e sul titolo di perfezionamento e sarà pubblicato nel MEDREG. GLN è la nuova denominazione del numero EAN (European Article Number). È consigliabile inserire il numero GLN nei comunicati e nella corrispondenza (p. es. carta da lettere, fatture, sito Internet). Tutti i medici in possesso di un diploma federale o riconosciuto sono attualmente registrati sotto un numero GLN. Ai medici provenienti da Stati membri dell'UE o dell'AELS che necessitano di un numero GLN e che non desiderano incorrere nell'obbligo di far riconoscere il proprio diploma per motivi legali (p.es. i medici assistenti), consigliamo ugualmente la procedura di riconoscimento, per il seguente motivo: l'assegnazione del titolo di perfezionamento federale può avvenire solo nel caso in cui il diploma sia stato riconosciuto.

I medici provenienti da Stati non membri dell'UE o non membri dell'AELS che necessitano di un numero GLN devono ugualmente inoltrare la relativa richiesta alla FMH.

\section{Quali sono i diritti e i doveri della FMH?}

La FMH - o in generale le organizzazioni professionali delle cinque professioni mediche universitarie - in base alla LPMed è tenuta a registrare i titoli di perfezionamento federali nel MEDREG. 
L'inserimento di qualifiche di diritto privato (titoli di perfezionamento, settori specialistici e certificati di capacità $F M H$ ) è, invece, facoltativo. Questa opzione è stata ancorata nell'ordinanza sul registro LPMed per volontà delle organizzazioni professionali. L'UFSP concorda sul fatto che una lista completa di tutte le qualifiche favorisce l'informazione dei pazienti ed è al contempo nell'interesse dei medici stessi. Per questo motivo la FMH pubblicherà nel MEDREG tutti i diplomi di perfezionamento di diritto privato.

La FMH dal canto suo trae vantaggio dal MEDREG poiché ha la possibilità di trasferire nella propria banca dati tutte le informazioni sulle nuove persone appartenenti alle professioni mediche che possiedono un diploma federale o estero riconosciuto.

\section{Quali dati non sono pubblici?}

- Data di nascita

- Luogo di attinenza

- $\mathrm{N}^{\circ}$ AVS

- Dati degni di particolare protezione

- Oneri e restrizioni

- Indirizzo e-mail

\section{Quali sono i diritti e i doveri del medico?}

In base alla LPMed e all'ordinanza sul registro LPMed il medico non è obbligato a registrare i dati direttamente nel MEDREG; infatti essi vengono registrati dall'UFSP, la FMH e le autorità cantonali a seconda delle competenze previste dall'ordinanza sul registro LPMed. II medico, nel caso svolga un'attività soggetta ad autorizzazione, sottosta tuttavia all'obbligo di notifica alle autorità cantonali preposte al rilascio delle autorizzazioni. A tale proposito, alcuni Cantoni hanno chiesto ai propri medici di collaborare nell'attuale fase di correzione e aggiornamento dei dati, poiché i loro dati risultano incompleti o non più attuali. A partire dal $1^{\circ}$ gennaio 2010 si potranno tuttavia comunicare gli eventuali cambiamenti relativi all'ambito professionale (p.es. cessione dello studio medico, cessione temporanea dell'attività in studio, trasferimento, ecc.) attraverso il MEDREG (per l'indirizzo v. in basso). I titoli di perfezionamento federali e i diplomi di perfezionamento FMH sono costantemente aggiornati dalla stessa FMH.

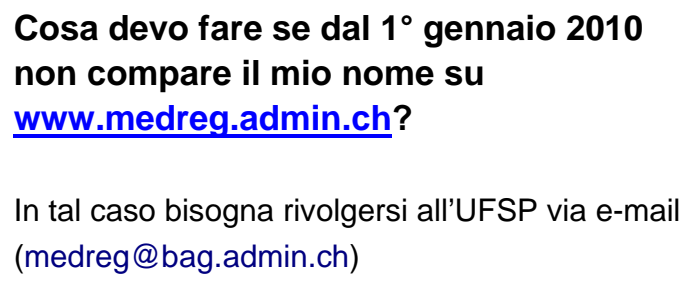

\section{Come si ottiene l'accesso al MEDREG?}

Da subito si può richiedere un nome utente con password all'indirizzo https://www.medreg.admin.ch/Anmelden.aspx . I dati di accesso saranno comunicati durante il $1^{\circ}$ trimestre 2010. Essi consentono di visionare tutte le informazioni registrate e di richiederne la rettifica per via elettronica nel caso esse siano incomplete o scorrette. Non appena la domanda di mutazione sarà inoltrata, l'organo competente verrà informato sulle modifiche desiderate. Qualora la domanda venga approvata, dopo essere stata esaminata, tutte le parti compartecipanti al progetto (autorità cantonali preposte al rilascio delle autorizzazioni, UFSP, organizzazione professionale) saranno informate contemporaneamente. In aggiunta, il pubblico avrà accesso al nuovo indirizzo dello studio medico, per esempio. II medico ha anche il diritto di richiedere all'UFSP la visione dei suoi dati personali degni di particolare protezione, qualora ve ne siano. 
Per i membri della FMH è previsto l'accesso diretto al MEDREG via myFMH. I membri saranno informati a tempo debito sulla realizzazione di tale accesso.

\section{In breve}

- II MEDREG è una banca dati volta principalmente all'informazione e alla protezione dei pazienti.

- La banca dati centralizzata agevola la collaborazione tra le diverse parti e aumenta soprattutto la trasparenza tra le autorità cantonali.

- Tutti i medici che sono in possesso di un'autorizzazione al libero esercizio della professione devono essere registrati nel MEDREG.

- La LPMed obbliga le autorità cantonali competenti a segnalare ogni concessione, rifiuto e modifica dell'autorizzazione al libero esercizio della professione, ogni restrizione e ogni provvedimento disciplinare.

- II registro delle professioni mediche non è una nuova banca dati di riferimento che autorizzi il conteggio di prestazioni in base all'assicurazione obbligatoria delle cure medico-sanitarie (AOMS).

- Un'iscrizione mancante o lacunosa se si è in possesso di un'autorizzazione all'esercizio della professione quindi non costituisce un motivo valido per cui la professione non possa essere svolta con tutti i rispettivi diritti e doveri (ciò che conta è l'autorizzazione cantonale all'esercizio della professione). È tuttavia consigliabile comunicare immediatamente eventuali correzioni.

\section{Devo realmente controllare di persona i miei dati inseriti nel MEDREG? \\ L'UFSP, i Cantoni e la FMH hanno riunito molto accuratamente i dati che avevano a disposizione. Si è pregati tuttavia di controllare i propri dati e di segnalare eventuali errori. Grazie.}

